\title{
Effectiveness and tolerability of transdermal buprenorphine patches: a multicenter, prospective, open-label study in Asian patients with moderate to severe chronic musculoskeletal pain
}

Do Heum Yoon ${ }^{1 *}$, Seong- -1 Bin $^{2}$, Simon Kin-Cheong Chan ${ }^{3}$, Chun Kee Chung ${ }^{4,5}$, Yong In ${ }^{6}$, Hyoungmin Kim Juan Javier Lichauco ${ }^{8}$, Chi Chiu Mok ${ }^{9}$, Young-Wan Moon ${ }^{10}$, Tony Kwun-Tung Ng ${ }^{11}$, Ester Gonzales Penserga ${ }^{12}$, Dong Ah Shin ${ }^{1}$, Dora You ${ }^{13}$ and Hanlim Moon ${ }^{13}$

\begin{abstract}
Background: We examined the effectiveness and tolerability of transdermal buprenorphine (TDB) treatment in real-world setting in Asian patients with musculoskeletal pain.

Methods: This was an open-label study conducted in Hong Kong, Korea, and the Philippines between June 2013 and April 2015. Eligible patients fulfilled the following criteria: 18 to 80 years of age; clinical diagnosis of osteoarthritis, rheumatoid arthritis, low back pain, or joint/muscle pain; chronic non-malignant pain of moderate to severe intensity (Box-Scale-11 [BS-11] pain score $\geq 4$ ), not adequately controlled with non-opioid analgesics and requiring an opioid for adequate analgesia; and no prior history of opioid treatment. Patients started with a $5 \mu \mathrm{g} / \mathrm{h}$ buprenorphine patch and were titrated as necessary to a maximum of $40 \mu \mathrm{g} / \mathrm{h}$ over a 6-week period to achieve optimal pain control. Patients continued treatment with the titrated dose for 11 weeks. The primary efficacy endpoint was the change in BS-11 pain scores. Other endpoints included patients' sleep quality and quality of life as assessed by the 8-item Global Sleep Quality Assessment Scale (GSQA) questionnaire and the EuroQol Group 5-Dimension Self-Report Questionnaire-3 Level version (EQ-5D-3 L), respectively. Tolerability was assessed by collecting adverse events.
\end{abstract}

Results: A total of 114 eligible patients were included in the analysis. The mean BS-11 score at baseline was 6.2 (SD 1.6). Following initiation of TDB, there was a statistically significant improvement in BS-11 score from baseline to visit 3 (least squares [LS] mean change: -2.27 [95\% Cl -2.66 to -1.87]), which was maintained till the end of the study (visit 7) (LS mean change: -2.64 [95\% -3.05 to -2.23]) ( $p<0.0001$ for both). The proportion of patients who rated sleep quality as 'good' increased from $14.0 \%$ at baseline to $26.9 \%$ at visit 6 . By visit 6, the mean EQ VAS score increased by 7.7 units (SD 17.9). There were also significant improvements in patients' levels of functioning for all EQ-5D-3 $L$ dimensions from baseline at visit 6 ( $p<0.05$ for all). Seventy-eight percent of patients reported TEAEs and $22.8 \%$ of patients discontinued due to TEAEs. TEAEs were generally mild to moderate in intensity (96.5\%).

* Correspondence: ydoheum@yuhs.ac; shindongah@me.com

'Department of Neurosurgery, Spine and Spinal Cord Institute, Yonsei

University College of Medicine, Severance Hospital, 134 Shinchon-dong

Seodaemun-gu, Seoul 120-752, South Korea

Full list of author information is available at the end of the article 
(Continued from previous page)

Conclusions: TDB provides effective pain relief with an acceptable tolerability profile over the 11-week treatment period in Asian patients with chronic musculoskeletal pain. More studies are needed to examine the long-term efficacy and safety of TBD treatment in this patient population.

Trial registration: ClinicalTrials.gov NCT01961271. Registered 7 October 2013 (retrospectively registered; first patient was enrolled on 28 June 2013 and last patient last visit date was 26 Apr 2015).

Keywords: Transdermal buprenorphine, Asian, Chronic non-malignant pain, Musculoskeletal, Pain score, Quality of life, Sleep quality, Effectiveness, Tolerability

\section{Background}

Musculoskeletal disorders are among the most common causes of chronic non-malignant pain in adults. Unrelieved pain has significant physiological and psychological impact on patients, affecting their daily quality of life as well as increasing their financial burden [1]. A comprehensive approach encompassing pharmacological and physical therapies, tailored to the individual needs of patients, is needed to reduce pain, promote functional recovery, and improve overall quality of life [2].

Systematic reviews examining evidence on the use of opioid analgesics for the treatment of chronic nonmalignant pain suggested these are efficacious in treating pain of various etiologies [3, 4]. However, opioid treatment is associated with typical opioid-induced side effects and potential risk of opioid abuse which can lead to addiction, severe respiratory depression, or even death [3-5]. Guidelines recommend the use of opioid analgesics for the treatment chronic non-malignant pain in patients whose pain persists despite optimized non-opioid treatment. They stress the importance of careful selection of patients who are not at risk of opioid abuse or diversion and ongoing monitoring, together with balancing the goal of achieving pain relief with the risks for opioid abuse or addiction when prescribing treatment [6-8]. Opioid formulations with extended-release (ER) and tamper-resistant properties offer the advantage of achieving analgesia while minimizing risks for opioid abuse or addiction [9-11]. Unlike immediate-release (IR) formulations, ER formulations provide relatively consistent and prolonged plasma drug levels with fewer peak-to-trough fluctuations and lower peak plasma concentration. This results in prolonged analgesia with less frequent dosing and reduced risks of opioid overdose, respiratory depression, and opioid addiction. In addition, the longer time to peak plasma concentration and tamper-resistant features make the formulations less desirable for abuse compared with IR formulations [9-11].

Transdermal buprenorphine (TDB) patches (marketed under different names depending on the country: Norspan ${ }^{\circ}$, Sovenor ${ }^{\circ}$, Butrans ${ }^{\circ}$, or Restiva ${ }^{\circ}$ ), in three dose strengths: $5 \mu \mathrm{g} / \mathrm{h}, 10 \mu \mathrm{g} / \mathrm{h}$, or $20 \mu \mathrm{g} / \mathrm{h}$, have been developed [12]. It is licensed for the treatment of moderate to severe chronic pain that do not respond to non-opioid analgesics [13-15]. Its active ingredient, buprenorphine, is a potent opioid analgesic that acts primarily as a partial agonist at the $\mu$ opioid receptor [16]. TDB offers several advantages over typical full $\mu$-opioid receptor agonists in treating chronic pain. First, its unique partial agonist activity induces a ceiling effect for respiratory depression but not for analgesia, resulting in a reduced risk for this potentially fatal adverse event compared to other full opioid agonists $[17,18]$. Next, TDB has a lower propensity for opioid abuse or addiction than typical full opioid agonists $[9,17]$ and its transdermal matrix makes it difficult to extract the substance for nonmedical use [10]. In addition, unlike most full opioid agonists which are eliminated primarily in urine, buprenorphine is mainly excreted through the feces and does not accumulate in the body. This makes it more suitable and convenient to use than full opioid agonists because it does not require special dose adjustments in patients with compromised renal function, such as the elderly or renal patients [18-21]. Next, TDB releases a steady and continuous dose of buprenorphine over a period of up to seven days which confers the convenience of once-weekly dosing. Given that prescribing of analgesics in patients with musculoskeletal disorders may be complicated by comorbid conditions and polypharmacy, the extended analgesia duration offered by TDB allows for less frequent dosing and reduces pill burden compared with other oral opioid agonists. This may help improve patient acceptability and adherence. Besides these, TDB can be used by patients who have difficulty swallowing, or have gastrointestinal disorders, or preexisting nausea and vomiting and are unable to take oral opioid analgesics [22].

TDB has demonstrated good efficacy and an acceptable tolerability profile in patients with chronic non-malignant pain in randomized controlled trials [23-25]. Pain intensity and sleep disturbance were considerably reduced and patients experienced improved physical function and quality of life after treatment. TDB was tolerated by the majority of the patients in these studies [23-25]. TDB was noted to be non-inferior to other opioid analgesics in reducing pain. Apart from application site reactions that were typical of transdermal delivery systems, TDB has an $\mathrm{AE}$ profile that is comparable with the other opioid 
analgesics [23, 25]. However, real-world data on treatment of chronic pain with TDB is limited [26]. To date, there are no multinational studies examining the use of TDB in Asian patients with chronic non-malignant pain. This study aimed to assess the effectiveness and tolerability of TDB in real-life clinical settings in Asian patients who were suffering from moderate to severe musculoskeletal pain.

\section{Methods}

\section{Study design}

This was a prospective, multicenter, open-label, singlearm study conducted in 16 hospital sites across three countries or territories in Asia (Hong Kong, Korea, and the Philippines) between June 2013 and April 2015. The study comprised a screening and baseline visit (visit 1) to assess patient eligibility and collect baseline data, followed by a titration period of up to six weeks, during which patients received the study medication and their dose was adjusted as necessary to achieve optimal pain control. Patients then entered an 11-week treatment period and attended a follow-up visit two weeks after completion of treatment. The study consisted of at least seven scheduled visits at specified intervals (Fig. 1).

\section{Patients}

Inclusion criteria were as follows: (i) $18-80$ years of age; (ii) clinical diagnosis of osteoarthritis, rheumatoid arthritis, low back pain, or joint/muscle pain; and (iii) chronic moderate to severe pain [defined as a score of $\geq 4$ on the Box Scale-11 (BS-11) pain scale], not adequately controlled with non-opioid analgesics and requiring an opioid for adequate analgesia. Exclusion criteria included the following: (i) pregnant or lactating females; (ii) females of childbearing potential who were not willing to use appropriate contraception during the study; (iii) current diagnosis or history of cancer (except basal cell carcinoma); (iv) previous surgery or required surgery; (v) history of alcohol or drug abuse, or behaviors suggestive of addiction or substance abuse; (vi) presence of any contraindication to the study medication; (vii) other chronic conditions that required frequent analgesic therapy; (viii) history of allergy to analgesic agents; (ix) history of prior treatment with study medication; (x) history of opioid treatment; (xi) current or history of steroid treatment; and (xii) deemed unsuitable for participation by the study physician.

\section{Study treatment}

7-day TDB patches, available in three dose strengths: $5 \mu \mathrm{g} / \mathrm{h}, 10 \mu \mathrm{g} / \mathrm{h}$, and $20 \mu \mathrm{g} / \mathrm{h}$, were used in this study. Patients started with a $5 \mu \mathrm{g} / \mathrm{h}$ buprenorphine patch at the baseline visit and were titrated as necessary to a maximum of $40 \mu \mathrm{g} / \mathrm{h}$ over a 6-week period to achieve optimal pain relief. Dose titration and patch application were carried out according to the instructions stated in the local summary of product characteristics or patient information leaflet [13-15]. Patients were prescribed standard recommended doses of rescue analgesics as needed. Antiemetics and laxatives were also permitted, if required. The optimal buprenorphine dose was determined at the physician's discretion based on patient's need for supplemental pain relief and analgesic response to the patch. Patients wore the patch continuously for 7 days before titration to the next dose was considered;

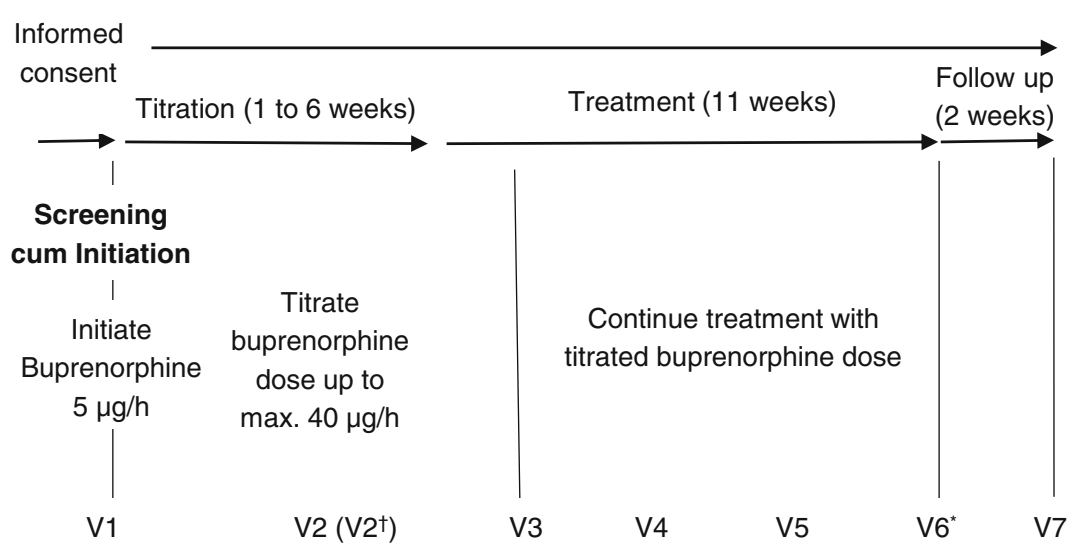

Visit schedule: V2/V2 ${ }^{\dagger}(\mathrm{V} 1+$ up to 6 weeks); V3 (1 week \pm 3 days after V2); V4 (2 weeks \pm 3 days after V3); V5 ( 4 weeks \pm 3 days after V4); V6 ( 4 weeks \pm 3 days after V5); V7 (2 weeks \pm 3 days after $V 6$ )

V2 ${ }^{\dagger}$ : Subsequent optional titration visits

"Patients could choose to continue on transdermal buprenorphine treatment, subjected to their physicians' approval.

Fig. 1 Study design 
however, the dose could be increased earlier, after three days of application if patients did not experience adequate analgesia despite administration of rescue analgesics. Buprenorphine dose was increased gradually in increments of $5 \mu \mathrm{g} / \mathrm{h}$ either with a patch of higher dose strength or with a combination of patches. No more than two patches were applied at the same time and a new skin site was selected for application. Patients who achieved optimal pain control entered the treatment period, which lasted for 11 weeks. During this period, the physicians adjusted patients' doses as necessary to maintain optimal pain control. Patients stopped TDB treatment when they completed the 11-week treatment. However, they could choose to continue on TDB treatment, subjected to their physicians' approval.

\section{Study assessments}

Patients' demographics and disease characteristics were collected at visit 1 . Information relating to concomitant illness was collected throughout the study. Concomitant illness was defined as any medical condition, other than the primary condition, that occurred within the last five years prior to study entry or during the course of the study.

\section{Primary efficacy assessment}

The primary efficacy assessment was the BS-11 pain score. Before the start of treatment (visit 1) and at each subsequent visit, patients rated their pain level on the BS-11 scale, ranging from a scale of 0 (no pain) to 10 (unbearable pain) [27].

\section{Secondary efficacy assessments}

The secondary efficacy assessments were as follows:

1. Global Sleep Quality Assessment Scale (GSQA) Before treatment (visit1) and at the end of treatment (visit 6) and the follow-up visit (visit 7), patients evaluated their overall sleep quality, degree of sleep disturbance, and sleep duration using the 8-item GSQA scale questionnaire. The GSQA scale ranges from 0 to 10, with higher scores indicating a higher degree of sleep disturbance.

2. EuroQol Group 5-Dimension Self-Report Questionnaire-3 Level Version Survey (EQ-5D-3 L questionnaire)

Patients assessed their quality of life using the EQ-5D$3 \mathrm{~L}$ questionnaire at visits 1,6 , and 7 . The questionnaire includes the EQ-5D visual analogue scale (EQ VAS) where patients rated their overall health state on a scale ranging from 0 (worst imaginable health state) to 100 (best imaginable health state). It also includes the descriptive system comprising the following five dimensions: mobility, self-care, usual activities, pain or discomfort, and anxiety or depression where patients indicated their levels of functioning at one of the three levels: no problems, some problems, and extreme problems [28].

3. Global Impression of Change Assessment Both patients and physicians evaluated the change in overall pain condition since the initiation of treatment. They completed the Patient Global Impression of Change questionnaire [29] and the Physician Global Impression of Change questionnaire [30], respectively at visit 6 . The change was rated on a 7-point scale. A score of 1 indicates "very much improved", 2 "much improved", 3 "minimally improved", 4 "no change", 5 "minimally worse", 6 "much worse", and 7 "very much worse".

4. Concomitant rescue medication use Patients recorded their rescue medication intake in the patient diary throughout the study.

Local language version of all questionnaires was used in each country or territory.

\section{Safety assessments}

The physicians recorded all adverse events (AEs) that occurred during the study. Vital signs measurements and physical examination were performed at visits, 1, 6, and 7.

\section{Statistical analysis \\ Sample size estimation}

The sample size was estimated based on the results of previous studies [23-25]. Assuming a dropout rate of $20 \%$, it was calculated that at least 53 patients had to be recruited from each country to detect differences in BS-11 scores from baseline for each country at $90 \%$ statistical power and at the 5\% significance level. As recruiting was progressing more slowly than expected, it was decided to evaluate the overall change in pain scores for the combined study population to ensure sufficient power. Therefore, a total of 119 patients were recruited from the participating countries.

\section{Efficacy analysis}

The primary efficacy endpoint was the change in BS-11 pain scores between baseline and each visit. A linear mixed model-repeated measures analysis was performed to estimate the least squares (LS) mean change in scores from baseline and 95\% confidence intervals (CIs). Baseline score, visit, and country or territory were included as covariates in the model.

Secondary endpoints evaluated included: (i) GSQA overall sleep quality rating, sleep disturbance scores, and sleep duration; (ii) EQ VAS score and ratings for individual EQ-5D-3 L dimensions; (iii) patients' and physicians' 
Global Impression of Change scores; and (iv) prescribed concomitant rescue medications. The Bowker-McNemar test was used to compare patients' ratings for their overall sleep quality and individual ED-5D-3 L dimensions between visits. Parametric tests were performed where assumptions of normality were met. Otherwise, nonparametric tests were used. The Wilcoxon signed-rank test was used to compare patients' GSQA sleep disturbance scores and sleep duration between visits, and to compare patients' and physicians' Global Impression of Change scores. The paired t-test was used to compare EQ VAS scores between visits. Rescue medication use was summarized using descriptive statistics.

The intent-to-treat (ITT) population was the primary population for efficacy analysis. The efficacy data were analyzed using the per-protocol (PP) population to provide confirmation. The ITT population included eligible patients who received at least one dose of study medication and had pre- and at least one post-intervention assessment of efficacy variables. The PP population was defined as a subset of the ITT population who had completed all the visits without major protocol deviation or violation. All analyses were conducted using available data; no imputation was performed for missing data. A $p$-value of less than 0.05 was considered as statistically significant.

\section{Safety analysis}

Key safety endpoints evaluated included the incidence of treatment-emergent adverse events (TEAEs) and incidence of TEAEs leading to discontinuation, as well as changes in vital signs and physical examination parameters from baseline to visits 6 and 7. A TEAE was defined as any $\mathrm{AE}$ with an onset date on or after the first dose of TDB. Medications prescribed for prevention or for treatment of AEs associated with the study medication were also evaluated. Safety results were descriptively summarized for the safety population. The safety population included eligible patients who received at least one dose of study medication and had at least one safety follow up. All statistical analyses were performed using SAS software, version 9.3 (SAS Institute, Inc., USA).

\section{Results}

Patient demographics and baseline characteristics

The flow of patients through the study is shown in Fig. 2. One hundred and nineteen patients were enrolled into the study, five of whom did not meet the eligibility criteria and were excluded from the analysis. Among the remaining one hundred and fourteen eligible patients who received TDB treatment, 64 patients $(56.1 \%)$ completed the study and 50 (43.9\%) discontinued from the study. The reasons for discontinuation are summarized in Fig. 2. All 114 patients were included in the ITT population and safety population. The PP population included 63 patients who completed the study and had no major protocol deviation or violation.

Patients' demographics and characteristics at study entry are summarized in Table 1. Fifty-four percent of patients were from Korea while the rest were from Hong Kong and the Philippines (22.8\% each). The study population

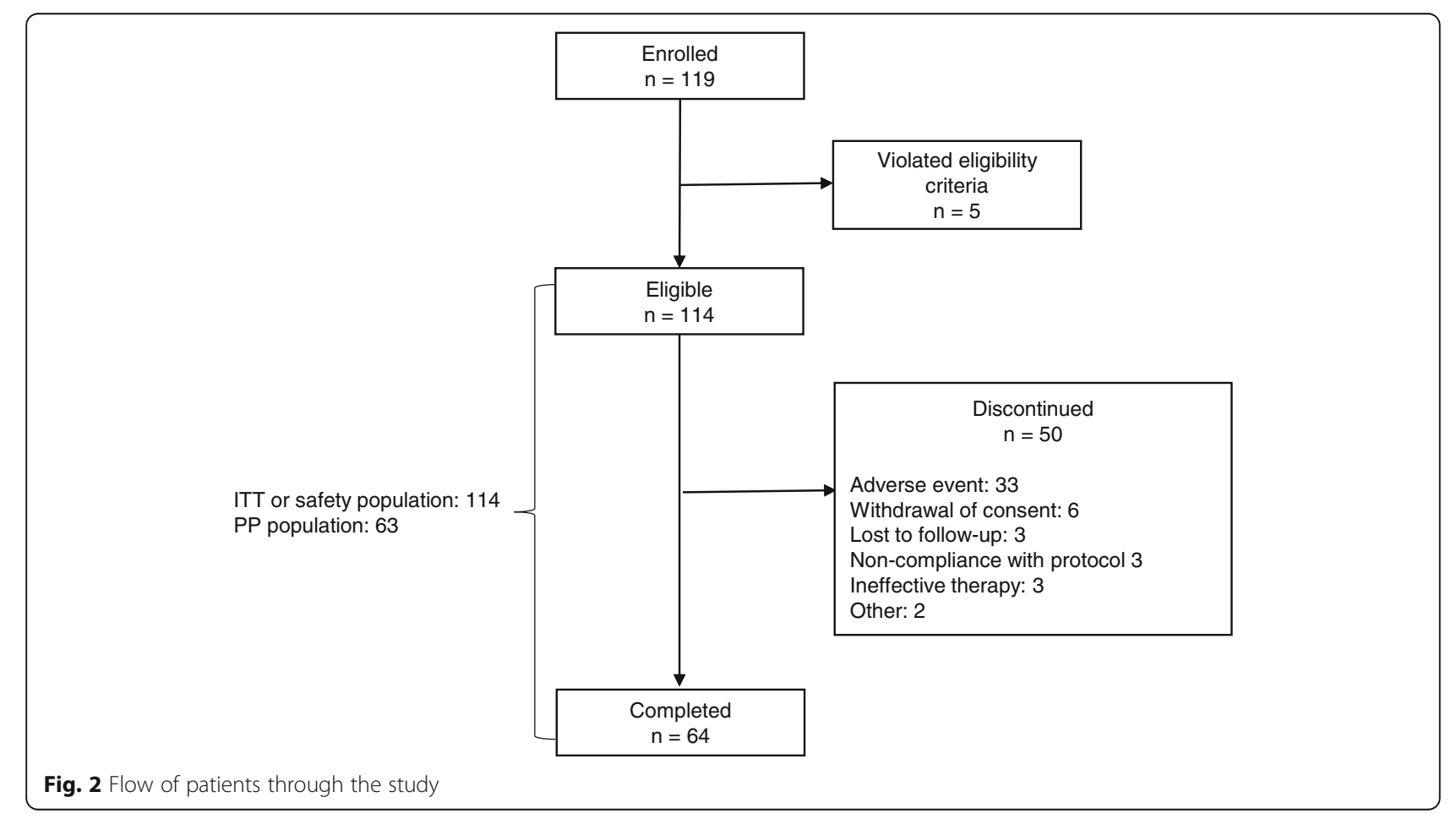


Table 1 Patient demographics and characteristics at study entry

\begin{tabular}{|c|c|c|c|c|}
\hline \multirow[t]{2}{*}{ Characteristics } & \multicolumn{4}{|c|}{ Safety population } \\
\hline & $\begin{array}{l}\text { Hong Kong } \\
(n=26)\end{array}$ & $\begin{array}{l}\text { Korea } \\
(n=62)\end{array}$ & $\begin{array}{l}\text { Philippines } \\
(n=26)\end{array}$ & $\begin{array}{l}\text { All } \\
(n=114)\end{array}$ \\
\hline Age (years), mean (SD) & $55.2(7.7)$ & $55.6(15.5)$ & $62.2(11.3)$ & $57.0(13.4)$ \\
\hline \multicolumn{5}{|l|}{ Gender, $n(\%)$} \\
\hline Male & $8(30.8)$ & $16(25.8)$ & $4(15.4)$ & $28(24.6)$ \\
\hline Female & $18(69.2)$ & $46(74.2)$ & $22(84.6)$ & $86(75.4)$ \\
\hline \multicolumn{5}{|l|}{ Causes of pain, $n(\%)$} \\
\hline Osteoarthritis & $6(23.1)$ & $29(46.8)$ & $20(76.9)$ & $55(48.3)$ \\
\hline Rheumatoid arthritis & $6(23.1)$ & $0(0.0)$ & $2(7.7)$ & $8(7.0)$ \\
\hline Low back pain & $9(34.6)$ & $32(51.6)$ & $2(7.7)$ & $43(37.7)$ \\
\hline Joint or muscle pain & $5(19.2)$ & $1(1.6)$ & $2(7.7)$ & $8(7.0)$ \\
\hline \multicolumn{5}{|c|}{${ }^{a}$ Concomitant illnesses, $n$ (\%) } \\
\hline Yes & $25(96.2)$ & $48(77.4)$ & $20(76.9)$ & $93(81.6)$ \\
\hline No & $1(3.8)$ & $14(22.6)$ & $6(23.1)$ & $21(18.4)$ \\
\hline
\end{tabular}

SD standard deviation

a Concomitant illness was defined as any medical condition, other than the primary condition, that occurred within the last five years prior to study entry or during the course of the study

was predominantly female (75.4\%), with a mean age of 57.0 (SD 13.4) years. Common causes of musculoskeletal pain were osteoarthritis (48.3\%) and low back pain (37.7\%). Sixty-two percent of patients had moderate pain (BS-11 score 4 to 6 ) and $38.1 \%$ had severe pain (BS-11 score $\geq 7$ ) at study entry. The majority of patients (81.6\%) reported at least one concomitant illness during the study period. Patients' demographics and characteristics varied across the countries or territories. The Philippines tended to have a higher mean age (62.2 [SD 11.3]) years and a higher proportion of female patients $(84.6 \%)$ than Hong Kong and Korea. In Hong Kong, patients reported a fairly even distribution of pain arising from a range of musculoskeletal conditions, with a trend towards higher frequency of low back pain (34.6\%). In Korea, low back pain (51.6\%) and osteoarthritis (46.8\%) were more common while osteoarthritis $(76.9 \%)$ was the most common cause of chronic pain in the Philippines. Hong Kong had the highest proportion of patients $(96.2 \%)$ who had concomitant illnesses across countries or territories.

\section{Exposure}

Patients received TDB for a median duration of 12.1 (range 0.3 to 19.1 ) weeks, at a median dose of 5.0 (5.0 to 40.0) $\mu \mathrm{g} / \mathrm{h}$ over the study period. All patients were initiated on TDB at a median dose of $5.0 \mu \mathrm{g} / \mathrm{h}$. The dose during titration was 5.0 (range 5.0 to 10.0 ) $\mu \mathrm{g} / \mathrm{h}$ at visit 2 $(n=100)$ and was titrated to 10.0 (range 5.0 to 40.0$) \mu \mathrm{g} / \mathrm{h}$ among those who required additional titration $(n=67)$. It decreased to 5.0 (range 5.0 to 40.0 ) $\mu \mathrm{g} / \mathrm{h}$ at visit 3 and was maintained at the same dose for the rest of the treatment period (Fig. 3). The most frequently prescribed dose over the study period was $5 \mu \mathrm{g} / \mathrm{h}$ (65.1\% of total prescriptions), followed by $10 \mu \mathrm{g} / \mathrm{h}$ (22.7\%), and $15 \mu \mathrm{g} / \mathrm{h}$ (7.4\%). Other doses were less frequently used: $20 \mu \mathrm{g} / \mathrm{h}(2.5 \%), 25 \mu \mathrm{g} / \mathrm{h}$ (1.0\%), $30 \mu \mathrm{g} / \mathrm{h}(0.4 \%)$, and $40 \mu \mathrm{g} / \mathrm{h}(1.0 \%)$.

\section{Efficacy}

The results of all efficacy analyses conducted in the ITT population were consistent with those of the PP analyses. Therefore, only results of the ITT population are presented here.

\section{Change in BS-11 pain score}

Figure 3 summarizes the change in BS-11 scores from baseline to the end of study in the ITT population. The mean BS-11 score at baseline was 6.2 (SD 1.6). Following initiation of TDB treatment, there was a statistically significant improvement in BS-11 score from baseline to visit 3 , which was maintained till the end of the study (visit 7) ( $p<0.0001$ for all). The corresponding LS mean change in score from baseline was -2.27 (95\% CI -2.66 to -1.87$)$ at visit 3 and $-2.64(95 \%-3.05$ to -2.23$)$ at visit 7 .

\section{Improvements in sleep quality and quality of life}

A marginally significant improvement $(p=0.054)$ in overall sleep quality assessment was observed at visit 6 . The proportion of patients rating sleep quality as 'good' increased from $14.0 \%$ at baseline to $26.9 \%$ at visit 6 . Patients reported a median sleep duration of 6.0 (range 2.0 to 9.0) hours per night over the seven days prior to the start of treatment. There was no clear improvement in sleep duration from baseline at visit 6. Patients reported minimal or low degree of sleep disturbance for all six variables at baseline (Table 2). By visit 6 , the median sleep disturbance scores for the sleep variables "trouble falling asleep" and "awakened by pain in the morning" improved significantly by one unit from baseline $(p<0.0001$ for both). There were no clear improvements in scores for the other sleep variables at visit 6 (Table 2).

The mean EQ VAS score at baseline was 59.6 (SD 15.9). Patients' overall health state improved significantly from baseline $(p<0.0001)$ after treatment. By visit 6 , the mean EQ VAS score had increased by 7.7 units (SD 17.9). There were also significant improvements in patients' levels of functioning for all EQ-5D-3 L dimensions from baseline at visit 6 ( $p<0.05$ for all) (Fig. 4). The proportion of patients who indicated they had no problem with each dimension increased from baseline to visit 6: mobility (from $29.0 \%$ to $54.8 \%$ ), self-care (from $60.5 \%$ to $80.7 \%$ ), usual activities (from $29.0 \%$ to $48.4 \%$ ), pain or discomfort (from $6.1 \%$ to $20.4 \%$ ), anxiety or depression (from $36.8 \%$ to $54.8 \%$ ) (Fig. 4 ). 


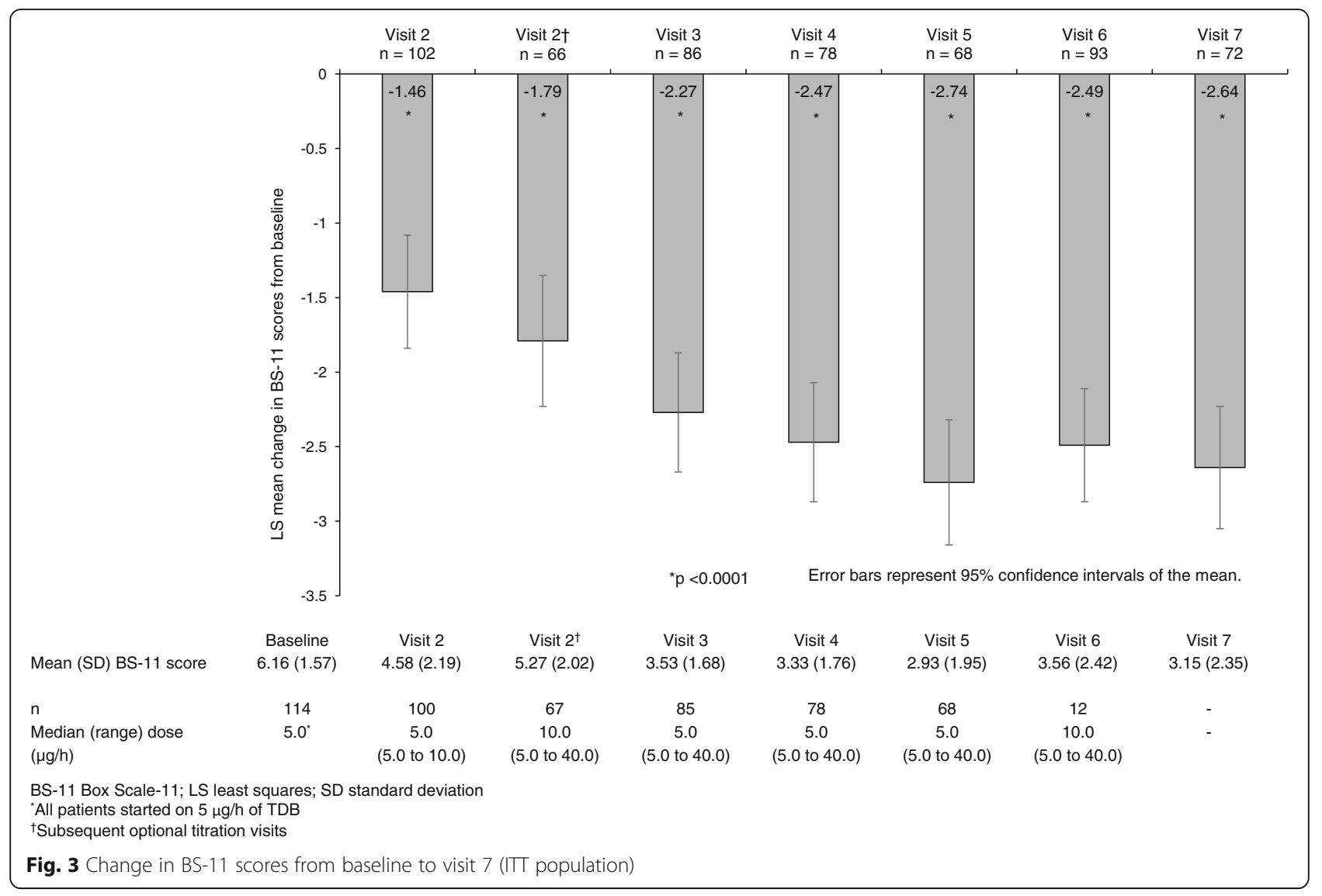

Table 2 Improvements in GSQA scores from baseline to visit 6

\begin{tabular}{|c|c|c|c|c|c|c|c|}
\hline \multicolumn{2}{|c|}{ GSQA Variables } & \multicolumn{6}{|l|}{ ITT population $(n=114)$} \\
\hline & & $\begin{array}{l}\text { Trouble falling } \\
\text { asleep }\end{array}$ & $\begin{array}{l}\text { Need pain medication } \\
\text { to sleep }\end{array}$ & $\begin{array}{l}\text { Need sleep medication } \\
\text { to sleep }\end{array}$ & $\begin{array}{l}\text { Awakened by } \\
\text { pain at night }\end{array}$ & $\begin{array}{l}\text { Awakened by pain } \\
\text { in the morning }\end{array}$ & $\begin{array}{l}\text { Effect of pain on } \\
\text { partner's sleep }\end{array}$ \\
\hline \multirow[t]{4}{*}{ Baseline } & $n$ & 114 & 114 & 114 & 114 & 114 & 86 \\
\hline & $\begin{array}{l}\text { Median } \\
\text { (range) }\end{array}$ & 3.00 (0.00 to 10.00$)$ & 1.00 (0.00 to 10.00$)$ & 0.00 (0.00 to 10.00$)$ & 2.00 (0.00 to 10.00$)$ & $2.00(0.00$ to 10.00 & 1.00 (0.00 to 10.00$)$ \\
\hline & Mean (SD) & $3.73(3.18)$ & $2.30(2.96)$ & $0.91(2.13)$ & 3.09 (3.19) & $2.96(3.41)$ & $2.71(3.42)$ \\
\hline & Missing & 0 & 0 & 0 & 0 & 0 & 28 \\
\hline \multirow[t]{4}{*}{ Visit 6} & $n$ & 93 & 93 & 93 & 93 & 93 & 80 \\
\hline & $\begin{array}{l}\text { Median } \\
\text { (range) }\end{array}$ & $1.00(0.00$ to 10.00$)$ & $0.00(0.00$ to 10.00$)$ & $0.00(0.00$ to 10.00$)$ & $1.00(0.00$ to 10.00 & $2.00(0.00$ to 10.00 & $0.00(0.00$ to 10.00 \\
\hline & Mean (SD) & $2.34(2.85)$ & $1.74(2.86)$ & $0.91(2.13)$ & $2.30(2.89)$ & $2.96(3.41)$ & $1.69(2.60)$ \\
\hline & Missing & 21 & 21 & 21 & 21 & 21 & 34 \\
\hline \multirow[t]{5}{*}{${ }^{\mathrm{a} C h a n g e}$} & $n$ & 93 & 93 & 93 & 93 & 93 & 70 \\
\hline & ${ }^{\dagger} p$-value & $<0.0001$ & 0.001 & 0.578 & 0.004 & $<0.0001$ & 0.007 \\
\hline & $\begin{array}{l}\text { Median } \\
\text { (range) }\end{array}$ & $-1.00(-10.00$ to 5.00$)$ & $0.00(-10.00$ to 7.00$)$ & $0.00(-10.00$ to 10.00$)$ & $0.00(-10.00$ to 7.00$)$ & $-1.00(-10.00$ to 7.00$)$ & $0.00(-10.00$ to 5.00$)$ \\
\hline & Mean (SD) & $-1.68(3.15)$ & $-0.86(2.83)$ & $-0.14(2.85)$ & $-1.04(3.28)$ & $-1.53(3.27)$ & $-1.09(3.08)$ \\
\hline & Missing & 21 & 21 & 21 & 21 & 21 & 44 \\
\hline
\end{tabular}




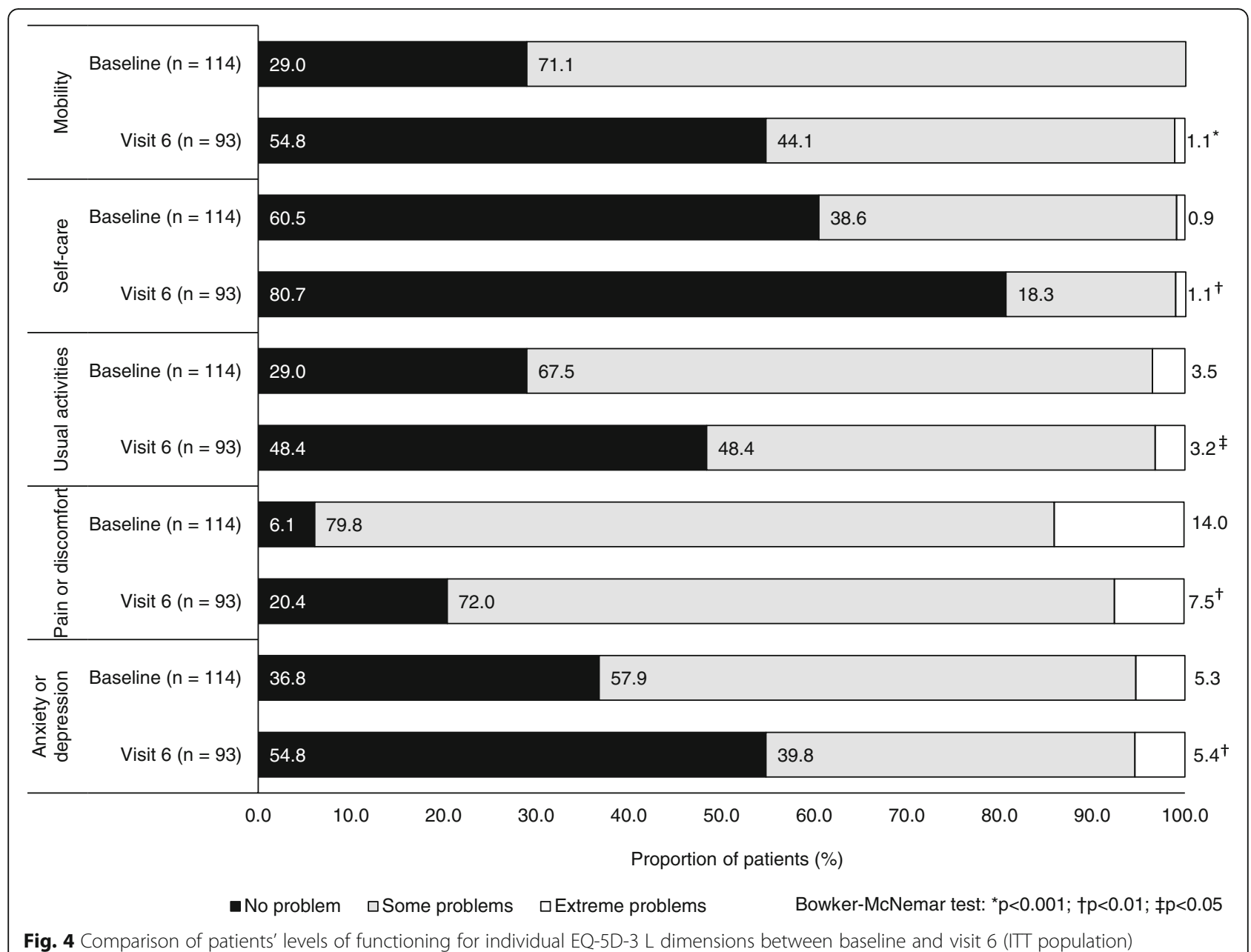

\section{Patient and physician global assessment of pain relief}

There was no significant difference between patients' and physicians' Global Impression of Change scores at visit $6(p=0.248)$. The median Global Impression of Change score was 3.0 (range 1.0 to 5.0) for both patients and physicians, indicating perceived improvement in overall pain condition at the end of TDB treatment.

\section{Rescue medication use}

Only $22.8 \%$ of patients required rescue medications during the study period. The most frequently prescribed rescue medications were acetaminophen (56.1\% of total prescriptions), followed by diclofenac (23.6\%). Other medications were less frequently used $(0.8-7.3 \%)$.

\section{Safety}

Overall, eighty-nine patients $(78.1 \%)$ reported TEAEs, most of which were mild to moderate in intensity (96.5\%). The most common TEAEs reported during the study were nausea (39.5\%) and constipation (31.6\%), followed by dizziness $(27.2 \%)$, somnolence (19.3\%), vomiting (16.7\%), headache $(8.8 \%)$, pruritus $(7.9 \%)$, and -application site reactions (6.1\%). Sixty-eight percent of patients had TEAEs that were assessed to be related to the study medication by the physician. One serious TEAE was reported during the course of the study. A patient experienced a hypertensive crisis; the event was moderate in severity but was not considered related to the study medication. The incidence of TEAEs leading to discontinuation was $22.8 \%$, most of the events were mild-moderate in intensity (92.9\%). TEAEs that frequently led to discontinuation of the study medication included nausea (11.4\%), dizziness (7.9\%), and vomiting (5.3\%).

Table 3 summarizes the incidence of TEAEs and common TEAEs, as well as the practice pattern in AE management of TDB treatment in the participating countries or territories. The incidence of TEAEs varied across countries or territories: Korea had a lower incidence of TEAEs (69.4\%) than the Philippines (80.8\%) and Hong Kong (96.2\%). A similar trend was also observed for discontinuation due to TEAEs, with a lower incidence in Korea (14.5\%) than the Philippines (26.9\%) and Hong Kong (38.5\%). Physicians more frequently prescribed medications to treat AEs that occurred during 
Table 3 Incidence of TEAEs and common TEAEs, and AE management of TDB treatment

\begin{tabular}{|c|c|c|c|c|}
\hline & \multicolumn{4}{|c|}{ Safety population } \\
\hline & $\begin{array}{l}\text { Hong Kong } \\
(n=26) \\
n(\%) \\
\end{array}$ & $\begin{array}{l}\text { Korea } \\
(n=62) \\
n(\%)\end{array}$ & $\begin{array}{l}\text { Philippines } \\
(n=26) \\
n(\%)\end{array}$ & $\begin{array}{l}\text { All } \\
(n=114) \\
n(\%)\end{array}$ \\
\hline Incidence of TEAEs & $25(96.2)$ & $43(69.4)$ & $21(80.8)$ & $89(78.1)$ \\
\hline $\begin{array}{l}\text { TEAEs leading to } \\
\text { discontinuation }\end{array}$ & $10(38.5)$ & $9(14.5)$ & $7(26.9)$ & $26(22.8)$ \\
\hline \multicolumn{5}{|l|}{${ }^{\mathrm{a} C o m m o n}$ TEAEs } \\
\hline Nausea & $11(42.3)$ & $27(43.6)$ & $7(26.9)$ & $45(39.5)$ \\
\hline Constipation & $10(38.5)$ & $20(32.3)$ & $6(23.1)$ & $36(31.6)$ \\
\hline Dizziness & $13(50.0)$ & $9(14.5)$ & $9(34.6)$ & $31(27.2)$ \\
\hline Somnolence & $9(34.6)$ & $8(12.9)$ & $5(19.2)$ & $22(19.3)$ \\
\hline Vomiting & $9(34.6)$ & $4(6.5)$ & $6(23.1)$ & $19(16.7)$ \\
\hline Headache & $4(15.4)$ & $2(3.2)$ & $4(15.4)$ & $10(8.8)$ \\
\hline Pruritus & $4(15.4)$ & $4(6.5)$ & $1(3.9)$ & $9(7.9)$ \\
\hline $\begin{array}{l}\text { Application site } \\
\text { reactions }\end{array}$ & $3(11.5)$ & $0(0.0)$ & $4(15.4)$ & $7(6.1)$ \\
\hline \multicolumn{5}{|c|}{${ }^{\mathrm{b}} \mathrm{AE}$ management of TDB treatment } \\
\hline $\begin{array}{l}\text { Received medications } \\
\text { for prevention and/or } \\
\text { treatment of common } \\
\text { AEs }\end{array}$ & $10(38.5)$ & $26(41.9)$ & $5(19.2)$ & $41(36.0)$ \\
\hline${ }^{\mathrm{C}}$ Prevention & $2(7.7)$ & $15(24.2)$ & $0(0.0)$ & $17(14.9)$ \\
\hline Antiemetics & $1(3.9)$ & $15(24.2)$ & $0(0.0)$ & $16(14.0)$ \\
\hline Laxatives & $1(3.9)$ & $0(0.0)$ & $0(0.0)$ & $1(0.88)$ \\
\hline${ }^{\mathrm{d}}$ Treatment & $8(30.8)$ & $14(22.6)$ & $5(19.2)$ & $27(23.7)$ \\
\hline Antiemetics & $2(7.7)$ & $11(17.7)$ & $2(7.7)$ & $15(13.2)$ \\
\hline Laxatives & $5(19.2)$ & $3(4.8)$ & $5(19.2)$ & $13(11.4)$ \\
\hline Antivertigo agents & $3(11.5)$ & $0(0.0)$ & $0(0.0)$ & $3(2.6)$ \\
\hline
\end{tabular}

$A E$ adverse event, $T D B$ transdermal buprenorphine, TEAE treatment-emergent adverse events

${ }^{a}$ Occurring in $\geq 5 \%$ of the overall safety population

${ }^{b}$ Patients may receive one or more medications for $A E$ management

CPrescribed on the same date as the start date of TDB treatment

${ }^{\mathrm{d}}$ Prescribed after the start of TDB treatment

TDB treatment than to manage them prophylactically (Table 3). Only $14.9 \%$ of patients received prophylactic medications while $23.7 \%$ received medications for treatment of AEs. Antiemetics were the most common preemptive treatment prescribed while both antiemetics and laxatives were commonly prescribed to treat AEs. Of note, physicians in Korea, the country with the lowest incidence of TEAEs, tended to prescribe medications both to prevent $(24.2 \%)$ and treat (22.6\%) AEs. In contrast, physicians in the Philippines and Hong Kong tended to prescribe medications to treat AEs (19.2\% and 30.8\%, respectively) rather than to prevent their occurrence $(0.0 \%$ and $7.7 \%$, respectively) (Table 3 ).

There were no clinically significant changes in vital signs or physical examination results between baseline and the end of the study.

\section{Discussion}

This is the first multinational study of TDB treatment in Asian patients who were suffering from moderate to severe pain due to a range of musculoskeletal conditions. This study, reflecting real-world clinical practice, demonstrates that TDB provides effective pain relief and improves daily functioning and quality of life in Asian patients with chronic musculoskeletal pain after 11 weeks of treatment. Additionally, TDB demonstrates an acceptable tolerability profile in our Asian cohort over the treatment period, consistent with that observed in Caucasians.

In the present study, patients entered the study with an average BS-11 pain score of 6.2. After initiation of TDB, the mean BS-11 score decreased by 2.3 unit one week after the end of the titration period and the reduction was maintained till the end of the treatment period (LS mean change: -2.5 at visit 6). A reduction of approximately 2 units from baseline on the 11-point numeric rating scale has been demonstrated to correspond to a clinically meaningful improvement [31]. The improvements in BS-11 score observed in this study are therefore considered clinically relevant. In addition, the majority of patients did not require additional rescue medications for pain. Similar improvements were observed in an open-label, randomized trial conducted in UK [23]. In this trial, reduction in BS-11 score was sustained over the 11-week treatment period (mean reduction: 3.6 at end of titration and 4.0 at week 12) in patients with knee and/or hip osteoarthritis who received TDB plus oral paracetamol [23]. The benefits of TDB in providing analgesia in patients with chronic moderate to severe non-malignant pain are supported by results from other studies $[25,26]$. In a prospective study of younger patients and elderly patients with osteoarthritis-related pain in Sweden, Karlsson et al. observed significant reductions in BS-11 score in patients who were treated with TDB, regardless of age (LS mean change: -1.9 to -2.2 ). In addition, patients used less rescue medications after the start of the 12-week treatment. The mean number of tablets of rescue medication taken each day reduced from 5.2 to 5.7 at baseline to 2.1 to 2.8 during the treatment period [26]. In another open-label, randomized study comparing the efficacy and safety of TDB with prolonged-release tramadol tablets, similar improvement in BS-11 score (LS mean change: -2.3) was reported in Swedish patients after 12 weeks of treatment with TDB [25].

Significant differences in the metabolism and response to medicines have been documented among racial groups [32]. The prescribed TDB dose was lower in this study compared to other studies conducted in Caucasian patients $[24,26,33]$. The median dose for the last dose prescribed during the treatment period in this study was $5 \mu \mathrm{g} / \mathrm{h}$ while higher doses $(10-24 \mu \mathrm{g} / \mathrm{h})$ were prescribed in other studies 
$[24,26,33]$. This findings suggest that Asian patients may require lower doses of TDB than Caucasian patients. More studies are needed to establish the dose level of TDB for treatment of pain in Asian patients.

The goal of chronic pain management encompasses not only the relief of pain, but also maintaining or improving the daily functioning and quality of life of patients [2]. In the present study, in addition to reduction of pain intensity, patients' quality of life and levels of functioning improved at the end of treatment with TDB. The proportion of patients who rated sleep quality as 'good' on the 8-item GSQA scale increased after treatment. Patients reported improvement in overall health state (EQ VAS) after treatment. Improvements in patients' levels of functioning for all dimensions of the EQ-5D-3 L questionnaire were also observed. Similar results were observed in previous studies of TDB $[25,26]$. In the study by Karlsson et al., patients reported fewer nights of sleep disturbance. The EQ VAS score increased by an average of 6.8 at study completion [26]. In another study, the majority of patients (71\%) reported improved ratings in sleep quality at the end of treatment. Improvement in EQ VAS score at study completion was also observed [25].

Consistent with the decrease in pain scores in the present study, both physicians and patients rated TDB treatment as associated with improvement in the overall pain condition. Previous studies of TDB have also reported favorable physician and patient ratings for TDB treatment $[25,26]$. In the recent study by Karlsson et al., the majority of physicians (61 to $65 \%$ ) and patients (59 to 68\%) rated TDB as "good" or "very good" at relieving pain [26]. Similar high proportions were reported in another study (physicians: $72.1 \%$, patients: $64.7 \%$, respectively) [25].

No unexpected safety or tolerability concerns were raised in the present study. Although the incidence of AEs and the incidence of discontinuations due to AEs were high, these were not unexpected as the incidence rates were consistent with those reported in previous studies of TDB conducted in Caucasian patients (incidence of AEs: 86 to $91 \%$ and incidence of discontinuations: 15 to $35 \%$ ) [23-26]. As with previous studies, AEs were generally mild or moderate. The most common AEs reported were consistent with the AE profile of TDB in general [23-26]. Of note, the incidence of skin reactions reported in this study was lower than that reported in other studies (27 to $30 \%)[23,24]$. As with previous studies [23, 25, 26], no significant abnormalities in vital signs or physical examination parameters were reported during the study.

Clinical guidelines from the American Pain SocietyAmerican Academy of Pain Medicine and the European Association for Palliative Care (EAPC) recommend physicians to employ preemptive intervention or symptomatic management of AEs to minimize the common side effects of opioid therapy [34, 35]. In the present study, prescription of medications for prevention or treatment of common AEs of TDB treatment was uncommon despite the high incidence of AEs reported. Although guidelines recommend to routinely prescribe laxative for prophylaxis management of opioid-induced constipation [34, 35], prescription of prophylactic laxative treatment was low in this study. These findings indicate a significant gap between guideline recommendations and the actual practice in managing the side effects of opioid treatment in the participating countries or territories. The development of education and training strategies to improve physicians' knowledge and to raise their awareness on opioid treatment may lead to improvements in treatment adherence.

In the present study, the incidence of AEs and the incidence of discontinuations varied across the countries or territories. The incidence rates appeared consistent with the practice pattern in side effect management of TDB treatment in each country or territory. Lower incidence rates were observed in Korea where medications for management of common AEs were prescribed both prophylactically and after TDB treatment. In contrast, higher incidence rates were observed in Hong Kong and the Philippines where physicians tended to prescribe medications to treat $\mathrm{AE}$ rather than to prevent their occurrence. These findings highlight the importance of preemptive and active management of side effects of TDB treatment in supporting treatment adherence. While practice pattern in side effect management influence the incidence rates, it is possible that ethnic differences in the development of AEs may exist. More studies are needed to confirm if the varying $\mathrm{AE}$ profile across the countries or territories is due to ethnic differences. This will provide valuable information for physicians to properly manage the use of TDB for pain management in different ethnic groups.

Our findings need to be interpreted within the limitations of the study. First, the treatment duration of 11 weeks is relatively short, and whether the observed treatment effect continues in the longer term would require further research to confirm. Next, the GSQA questionnaire has not been formally validated. Nevertheless the use of a structured questionnaire to assess sleep disturbance and sleep quality ensured consistency in data collection before and after TDB treatment and minimized bias to some extent. Another limitation is that the study population comprised a heterogeneous mix of musculoskeletal conditions with distinct pathophysiological mechanisms. It is probable that some conditions responded better than others to the treatment. However, the sample size is too small to allow parsing of the results by musculoskeletal condition. Future studies in additional populations of patients are needed to evaluate the effect of TDB in different 
conditions. In addition, there is potential for bias due to the open-label study design and the lack of active control in this study which preclude evaluation of the extent to which the observed effect is caused by the treatment. Nonetheless, the present study closely reflects the real-life clinical setting and the results are more likely to translate effectively into real-world clinical practice.

\section{Conclusions}

Our study provides real-world clinical evidence on the effectiveness and tolerability of TDB in Asian patients with moderate to severe chronic pain. Treatment with TDB resulted in effective and sustained pain relief over the 11-week treatment period, accompanied by improvements in daily functioning and quality of life. The tolerability profile was as expected as previous studies of TDB. Our results indicate that TDB can be considered a suitable alternative treatment option to control non-malignant musculoskeletal pain. Studies on the long-term efficacy and safety of TDB treatment are required to further confirm these findings.

\section{Abbreviations}

AEs: Adverse events; BS-11: Box Scale-11; Cls: Confidence intervals; EAPC: European Association for Palliative Care; EQ VAS: EQ-5D visual analogue scale; EQ-5D-3 L questionnaire: EuroQol Group 5-Dimension SelfReport Questionnaire-3 Level Version Survey; GSQA: Global Sleep Quality Assessment Scale; ITT: Intent-to-treat; LS: Least squares; NICE: National Institute for Health and Care Excellence; NSAIDs: Nonsteroidal antiinflammatory drugs; PP: Per-protocol; SD: Standard deviation; TDB: Transdermal buprenorphine; TEAEs: Treatment-emergent adverse events

\section{Acknowledgements}

Statistical support was provided by Liang Shen and editorial support was provided by Hui Hwa Choo of Research2Trials Clinical Solutions Pte Ltd., Singapore and was funded by Mundipharma Pte Ltd., Singapore. $\bullet:$ NORSPAN, BUTRANS, and RESTIVA are registered trade marks of Mundipharma AG.

TM: SOVENOR is a trade mark of Mundipharma AG.

\section{Funding}

This study was funded by Mundipharma Pte Ltd., Singapore.

\section{Availability of data and materials}

A summary of the data generated during the current study are available in the ClinicalTrials.gov repository (registration number: NCT01961271), https:// clinicaltrials.gov/show/NCT01961271. The complete data are available from the authors upon reasonable request.

\section{Authors' contributions}

$H M$ and DY were involved in the conception and design of the study. DHY SIB, SKCC, CKC, YI, HK, JJL, CCM, YWM, TKTN, EGP, and DAS were involved in data acquisition. All authors were involved in the interpretation of data and in revising the manuscript critically for important intellectual content. All authors gave final approval of the version to be published.

\section{Ethics approval and consent to participate}

This study was approved by local ethics committees (ECs) and was conducted in accordance with the International Conference on Harmonization Good Clinical Practice guideline, the Declaration of Helsinki, and applicable regulatory requirements. All eligible patients were invited to participate in the study. The physicians provided patients with oral and written information describing the nature, purpose, possible risk and benefits of the study. All patients provided written informed consent prior to participation in the study.
The local ECs that approved the study included (i) the Institutional Review Board of the University of Hong Kong/Hospital Authority Hong Kong West Cluster, Kowloon Central/Kowloon East Cluster Research Ethics Committee, Joint Chinese University of Hong Kong-New Territories East Cluster (CUHKNTEC) Clinical Research Ethics Committee, and New Territories West (NTW) Cluster \& Research Ethics Committee in Hong Kong; (ii) Asan Medical Center Institutional Review Board, Samsung Medical Center Institutional Review Board, Seoul St. Mary's Hospital Institutional Review Board, Severance Hospital Institutional Review Board, and Seoul National University Hospital Institutional Review Board in Korea; and (iii) Makati Medical Center Institutional Review Board, University of the Philippines Manila Research Ethics Board (UPMREB), St. Luke's Institutional Ethics Review Committee (SL-IERC), and the University of Santo Tomas Hospital-Institutional Review Board (USTH-IRB).

\section{Consent for publication}

Not applicable.

\section{Competing interests}

DHY, SIB, SKCC, CKC, YI, HK, CCM, YWM, TKTN, EGP, DAS, and JJL have received research funding from Mundipharma Pte Ltd., Singapore. JJL has also served on the advisory board for Pfizer and Novartis and has been on speaker bureaus for Pfizer, Johnson \& Johnson, and Merck Sharp \& Dohme. DY is an employee of Mundipharma Pte Ltd., Singapore and HM is a former employee of Mundipharma Pte Ltd., Singapore.

\section{Publisher's Note}

Springer Nature remains neutral with regard to jurisdictional claims in published maps and institutional affiliations.

\section{Author details}

${ }^{1}$ Department of Neurosurgery, Spine and Spinal Cord Institute, Yonsei University College of Medicine, Severance Hospital, 134 Shinchon-dong Seodaemun-gu, Seoul 120-752, South Korea. ${ }^{2}$ Department of Orthopedic Surgery, Asan Medical Center, Seoul, South Korea. ${ }^{3}$ Pain Management Unit, Department of Anaesthesiology and Intensive Care, Prince of Wales Hospital, The Chinese University of Hong Kong, Hong Kong, SAR, China. ${ }^{4}$ Department of Neurosurgery, Seoul National University Hospital, Seoul National University College of Medicine, Seoul, South Korea. ${ }^{5}$ Department of Brain and Cognitive Science, Seoul National University College of Natural Science, Seoul, South Korea. ${ }^{6}$ Department of Orthopaedic Surgery, Seoul St. Mary's Hospital, College of Medicine, The Catholic University of Korea, Seoul, South Korea. ${ }^{7}$ Department of Orthopedic Surgery, Seoul National University Hospital, Seoul National University College of Medicine, Seoul, South Korea. ${ }^{8}$ Section of Rheumatology, Department of Medicine, St. Luke's Medical Center, Manila, Philippines. ${ }^{9}$ Department of Medicine, Tuen Mun Hospital, Hong Kong, SAR, China. ${ }^{10}$ Department of Orthopedic Surgery, Samsung Medical Center, Sungkyunkwan University School of Medicine, Seoul, South Korea. ${ }^{11}$ Pain Management Unit, Department of Anaesthesia and Intensive Care, Tuen Mun Hospital, Hong Kong, SAR, China. ${ }^{12}$ Section of Rheumatology, Department of Medicine, University of the Philippines College of Medicine-Philippine General Hospital, Manila, Philippines. ${ }^{13}$ Mundipharma Pte Ltd, Asia Square Tower 2, Singapore.

Received: 26 September 2016 Accepted: 9 July 2017

Published online: 04 August 2017

\section{References}

1. Croft P, Blyth FM, van der Windt D. Chronic pain as a topic for epidemiology and public health. In: Croft P, Blyth FM, van der Windt D, editors. Chronic pain epidemiology. Oxford University Press: Academic; 2010. p. 3-8.

2. Zhang W, Moskowitz RW, Nuki G, Abramson S, Altman RD, Arden N, et al. OARSI recommendations for the management of hip and knee osteoarthritis, part II: OARSI evidence-based, expert consensus guidelines. Osteoarthr Cartil. 2008;16:137-62.

3. Furlan AD. Opioids for chronic noncancer pain: a meta-analysis of effectiveness and side effects. Can Med Assoc J. 2006;174:1589-94.

4. Kalso E, Edwards JE, Moore AR, McQuay HJ. Opioids in chronic non-cancer pain: systematic review of efficacy and safety. Pain. 2004;112:372-80.

5. Ling W, Mooney L, Hillhouse M. Prescription opioid abuse, pain and addiction: clinical issues and implications. Drug and alcohol review. 2011;30:300-5. 
6. Dowell D, Haegerich TM, Chou R. CDC guideline for prescribing opioids for chronic pain-United States, 2016. JAMA. 2016;315:1624-45.

7. Busse JW, Craigie S, Juurlink DN, Buckley DN, Wang L, Couban RJ, et al. Guideline for opioid therapy and chronic noncancer pain. CMAJ. 2017;189:E659-66.

8. O'Brien T, Christrup LL, Drewes AM, Fallon MT, Kress HG, McQuay HJ, et. al. European pain federation position paper on appropriate opioid use in chronic pain management. Eur J Pain 2017;21:3-19.

9. Brennan MJ. Update on prescription extended-release opioids and appropriate patient selection. J Multidiscip Healthc. 2013;6:265-80.

10. Pergolizzi JV Jr, Scholten W, Smith KJ, Leighton-Scott J, Willis JC, Henningfield JE. The unique role of transdermal buprenorphine in the global chronic pain epidemic. Acta Anaesthesiol Taiwanica. 2015;53:71-6.

11. Likar R. Transdermal buprenorphine in the management of persistent pain safety aspects. Ther Clin Risk Manag. 2006;2:115-25.

12. Kress HG. Clinical update on the pharmacology, efficacy and safety of transdermal buprenorphine. Eur J of Pain. 2009;13:219-30.

13. Norspan ${ }^{\ominus}$ Package insert. Mundipharma (Hong Kong) Limited, Hong Kong, 2011.

14. Norspan ${ }^{\oplus}$ Summary of product characteristics. Mundipharma Korea Ltd., Korea 2013.

15. Norspan ${ }^{\oplus}$ Patient information leaflet. Mundipharma Distribution $\mathrm{GmbH}$ (Philippine Branch), Philippines, 2010.

16. Hoskin PJ, Hanks GW. Opioid agonist-antagonist drugs in acute and chronic pain states. Drugs. 1991;41:326-44.

17. Dahan A, Yassen A, Romberg R, Sarton E, Teppema L, Olofsen E, et. al. Buprenorphine induces ceiling in respiratory depression but not in analgesia. Br J Anaesth 2006;96:627-632.

18. Pergolizzi J, Aloisi AM, Dahan A, Filitz J, Langford R, Likar R, et al. Current knowledge of buprenorphine and its unique pharmacological profile. Pain Pract. 2010;10:428-50.

19. Al-Tawil N, Odar-Cederlof I, Berggren AC, Johnson HE, Persson J. Pharmacokinetics of transdermal buprenorphine patch in the elderly. Eur J Clin Pharmacol. 2013;69:143-9.

20. Filitz J, Griessinger N, Sittl R, Likar R, Schüttler J, Koppert W. Effects of intermittent hemodialysis on buprenorphine and norbuprenorphine plasma concentrations in chronic pain patients treated with transdermal buprenorphine. Eur J of Pain. 2006;10:743-8.

21. Pergolizzi J, Böger RH, Budd K, Dahan A, Erdine S, Hans G, et al. Opioids and the management of chronic severe pain in the elderly: consensus statement of an international expert panel with focus on the six clinically most often used World Health Organization step III opioids (buprenorphine, fentanyl, hydromorphone, methadone, morphine, oxycodone). Pain Pract. 2008:8:287-313.

22. Hans G, Robert D. Transdermal buprenorphine - a critical appraisal of its role in pain management. J Pain Res. 2009;2:117-34.

23. Conaghan PG, O'Brien CM, Wilson M, Schofield JP. Transdermal buprenorphine plus oral paracetamol vs an oral codeine-paracetamol combination for osteoarthritis of hip and/or knee: a randomised trial. Osteoarthr Cartil. 2011;19:930-8.

24. Gordon A, Rashiq S, Moulin DE, Clark AJ, Beaulieu AD, Eisenhoffer J, et al. Buprenorphine transdermal system for opioid therapy in patients with chronic low back pain. Pain Res Manag. 2010;15:169-78.

25. Karlsson M, Berggren A-C. Efficacy and safety of low-dose transdermal buprenorphine patches $(5,10$, and $20 \mu \mathrm{g} / \mathrm{h}$ ) versus prolonged-release tramadol tablets $(75,100,150$, and $200 \mathrm{mg})$ in patients with chronic osteoarthritis pain: a 12-week, randomized, open-label, controlled, parallelgroup noninferiority study. Clinl Ther. 2009;31:503-13.

26. Karlsson J, Soderstrom A, Augustini BG, Berggren AC. Is buprenorphine transdermal patch equally safe and effective in younger and elderly patients with osteoarthritis-related pain? Results of an age-group controlled study. Curr Med Res Opin. 2014;30:575-87.

27. Jensen MP, Karoly P, O'Riordan EF, Bland F Jr, Burns RS. The subjective experience of acute pain. An assessment of the utility of 10 indices. Clin J Pain. 1989;5:153-9.

28. The EuroQol Group. EuroQol-a new facility for the measurement of health-related quality of life. Health Policy. 1990;16:199-208.

29. Ferguson L, Scheman J. Patient global impression of change scores within the context of a chronic pain rehabilitation program. J Pain. 2009;10:573.

30. Busner J, Targum SD. The clinical global impressions scale: applying a research tool in clinical practice. Psychiatry (Edgmont). 2007;4:28-37.
31. Farrar JT, Young JP Jr, LaMoreaux L, Werth JL, Poole RM. Clinical importance of changes in chronic pain intensity measured on an 11-point numerical pain rating scale. Pain. 2001;94:149-58.

32. Burroughs VJ, Maxey RW, Levy RA. Racial and ethnic differences in response to medicines: towards individualized pharmaceutical treatment. J Natl Medl Assoc. 2002;94:1-26.

33. Gordon A, Callaghan D, Spink D, Cloutier C, Dzongowski P, O'Mahony W, et al. Buprenorphine transdermal system in adults with chronic low back pain: a randomized, double-blind, placebo-controlled crossover study, followed by an open-label extension phase. Clin Ther. 2010;32:844-60.

34. Chou R, Fanciullo GJ, Fine PG, Adler JA, Ballantyne JC, Davies P, et al. American pain society-American Academy of pain medicine opioids guidelines panel. Clinical guidelines for the use of chronic opioid therapy in chronic noncancer pain. J Pain. 2009;10:113-30.

35. Caraceni A, Hanks G, Kaasa S, Bennett Ml, Brunelli C, Cherny N, et al. Use of opioid analgesics in the treatment of cancer pain: evidence-based recommendations from the EAPC. The Lancet Oncology. 2012;13:e58-68.

\section{Submit your next manuscript to BioMed Central and we will help you at every step:}

- We accept pre-submission inquiries

- Our selector tool helps you to find the most relevant journal

- We provide round the clock customer support

- Convenient online submission

- Thorough peer review

- Inclusion in PubMed and all major indexing services

- Maximum visibility for your research

Submit your manuscript at www.biomedcentral.com/submit
Biomed Central 